\title{
Caffeine-Containing Energy Shots Cause Acute Impaired Glucoregulation in Adolescents
}

\author{
Jane Shearer $1,2, * \mathbb{C}$, Raylene A. Reimer ${ }^{1,2} \mathbb{1}$, Dustin S. Hittel ${ }^{1,3}$, Mackenzie A. Gault ${ }^{1,2,4}$, \\ Hans J. Vogel ${ }^{5}$ and Matthias S. Klein ${ }^{6, * \mathbb{D}}$ \\ 1 Department of Biochemistry and Molecular Biology, Faculty of Kinesiology, University of Calgary, \\ Calgary, AB T2N 4N1, Canada; reimer@ucalgary.ca (R.A.R.); dhittel@gmail.com (D.S.H.); \\ magault@ucalgary.ca (M.A.G.) \\ 2 Alberta Children's Hospital Research Institute, Alberta Children's Hospital, Calgary, AB T2N 4N1, Canada \\ 3 Discovery DNA Inc., Calgary, AB T2N 4N1, Canada \\ 4 Department of Pediatrics, Alberta Children's Hospital, Calgary, AB T2N 4N1, Canada \\ 5 Department of Biological Sciences, Faculty of Science, University of Calgary, Calgary, AB T2N 1N4, Canada; \\ vogel@ucalgary.ca \\ 6 College of Food, Agricultural and Environmental Sciences, The Ohio State University, \\ Columbus, $\mathrm{OH} 43210$, USA \\ * Correspondence: jshearer@ucalgary.ca (J.S.); klein.663@osu.edu (M.S.K.); \\ Tel.: +1-403-220-3431 (J.S.); +1-614-688-1851 (M.S.K.)
}

Received: 19 November 2020; Accepted: 14 December 2020; Published: 16 December 2020

\begin{abstract}
Caffeine-containing, nutritionally fortified energy shots are consumed at high rates by adolescents, yet little is known about their metabolic impact. The purpose of this study was to examine the consequences of small format, caffeinated energy shots on glucose metabolism and gastrointestinal hormone secretion in adolescents. Twenty participants aged 13-19 years participated in a double-blind, randomized cross-over study consisting of two trials separated by 1-4 weeks. Participants consumed a volume-matched caffeinated energy shot (CAF, $5 \mathrm{mg} / \mathrm{kg}$ ) or a decaffeinated energy shot (DECAF) followed by a $2 \mathrm{~h}$ oral glucose tolerance test. Blood samples were collected and area under the curve (AUC) calculated for glucose, insulin and gut and metabolic hormones. Consumption of CAF resulted in a $25 \%$ increase in glucose and a $26 \%$ increase in insulin area under the curve (AUC, $p=0.037 ; p<0.0001$ ) compared to DECAF. No impact on gut hormones was observed. To further characterize responses, individuals were classified as either slow or fast caffeine metabolizers based on an allele score. Glucose intolerance was greater in genetically fast vs. slow caffeine metabolizers and differences between groups were supported by distinct serum metabolomics separation. Consumption of caffeine-containing energy shots results in acute impaired glucoregulation in healthy adolescents as characterized by hyperinsulinemia following an oral glucose challenge.
\end{abstract}

Keywords: pediatrics; adolescent; adverse reaction; energy drink; metabolomics

\section{Introduction}

Caffeine-containing energy drinks and small format energy shots (CED) are marketed to reduce fatigue and increase energy and alertness [1]. Along with caffeine, these beverages typically contain large amounts of other active ingredients, including vitamins, amino acids, flavorings and antioxidant compounds. An estimated $74 \%$ of youth consume energy drinks with $16 \%$ consuming more than two a day [2]. It is well recognized that energy drinks may be especially harmful for children, whose lower body mass may expose them to caffeine concentrations above recommended limits. Children often experience adverse impacts of energy drinks to a greater extent than adults for this reason [3]. CED 
consumption is associated with childhood obesity [4,5], likely impairs insulin sensitivity [6] and causes sleep disturbances in adolescents $[7,8]$.

From a metabolic perspective, it is accepted that co-administration of a carbohydrate load in the presence of caffeine impairs whole body glucose disposal by $20-30 \%$ in adults [9]. Reductions in whole body glucose disposal have been shown during oral glucose tolerance tests (OGTT) and euglycemic-hyperinsulinemic clamps [10-12]. Caffeine-induced glucose intolerance occurs in a dose-dependent manner at concentrations as low as $1 \mathrm{mg}$ caffeine/kg body weight, with no apparent threshold [13]. Habitual consumption of caffeine does not appear to result in an adaptation to this response [14]. As the half-life of caffeine is in the range of 4-6 h, insulin action could be impaired for some hours after ingestion. Given these data, one serving of a typical energy drink or small format energy shot would be expected to not only exceed daily recommended limits for caffeine for children (Health Canada, $2.5 \mathrm{mg} / \mathrm{kg}$ ), but also impair insulin sensitivity for most of their waking hours. However, it must be acknowledged that there are genetic contributors to caffeine metabolism, and that individual responses may vary [15]. To examine the impact of CED on glucose regulation, adolescents consumed either a caffeine-containing energy shot $(5 \mathrm{mg} / \mathrm{kg})$ or its equivalent decaffeinated version $40 \mathrm{~min}$ prior to an oral glucose tolerance test. Additional assessments included gut and metabolic hormones known to influence glucoregulation as well as a preliminary analysis of genetic contributors to the observed responses.

\section{Materials and Methods}

\subsection{Participants}

This study was approved by the Conjoint Health Research Ethics Board at the University of Calgary (REB14-1093) and registered at ClinicalTrials.gov (NCT03512496). Subjects were recruited by convenience sampling through local advertising. Twenty (10 males, 10 females) adolescents aged 13-19 years without underlying medical conditions, supplement use, medication intake affecting glucose tolerance, oral contraceptive use and without phenylketonuria or known caffeine allergies were invited to participate.

\subsection{Test Beverages}

The composition of the decaffeinated beverage (5-h Energy Decaffeinated, DECAF) and the caffeine-containing beverage (5-h Energy Original, Regular Strength, CAF) are shown in Table 1. Both are sugar-free and administered as pre-determined mixtures in identical cups and of equivalent volumes, color and taste (both were sucralose-sweetened). Each subject consumed $5 \mathrm{mg} / \mathrm{kg}$ of caffeine, corresponding to one $57 \mathrm{~mL}$ (commercially available) serving for a typical 13-year-old ( $\sim 38 \mathrm{~kg}$ ).

Table 1. Ingredient profiles of the caffeine-containing CAF (5-h Energy original) and DECAF (5-h Energy Decaffeinated) shots.

\begin{tabular}{|c|c|c|c|c|c|}
\hline \multicolumn{3}{|c|}{ 5-h Energy Original (CAF, Regular Strength) } & \multicolumn{3}{|c|}{ 5-h Energy Decaf (DECAF) } \\
\hline Ingredient & Amount & \%Daily Value & Ingredient & Amount & \%Daily Value \\
\hline Vitamin B6 & $40 \mathrm{mg}$ & $2000 \%$ & Vitamin B6 & $40 \mathrm{mg}$ & $2000 \%$ \\
\hline Folic Acid & $400 \mathrm{mcg}$ & $100 \%$ & Folic Acid & $400 \mathrm{mcg}$ & $100 \%$ \\
\hline Vitamin B12 & $500 \mathrm{mcg}$ & $8333 \%$ & Vitamin B12 & $500 \mathrm{mcg}$ & $8333 \%$ \\
\hline Sodium & $18 \mathrm{mg}$ & $<1 \%$ & Sodium & $18 \mathrm{mg}$ & $<1 \%$ \\
\hline Niacin & $30 \mathrm{mg}$ & $150 \%$ & Niacin & $0 \mathrm{mg}$ & $0 \%$ \\
\hline Caffeine & $215 \mathrm{mg}$ & $\mathrm{n} / \mathrm{a}$ & Caffeine & $6 \mathrm{mg}$ & $\mathrm{n} / \mathrm{a}$ \\
\hline Energy Blend & $1870 \mathrm{mg}$ & $\mathrm{n} / \mathrm{a}$ & Energy Blend & $2009 \mathrm{mg}$ & $\mathrm{n} / \mathrm{a}$ \\
\hline \multirow{2}{*}{\multicolumn{3}{|c|}{$\begin{array}{l}\text { (Taurine, glucuronic acid, malic acid, N-Acetyl } \\
\text { L-tyrosine, L-phenylalanine, caffeine, citicoline) }\end{array}$}} & \multirow{2}{*}{\multicolumn{3}{|c|}{$\begin{array}{l}\text { (Taurine, choline, glucuronic acid, N-Acetyl } \\
\text { L-tyrosine, L-Phenylalanine, malic acid, caffeine) }\end{array}$}} \\
\hline & & & & & \\
\hline \multicolumn{3}{|c|}{$\begin{array}{l}\text { Other Ingredients: Purified water, natural and } \\
\text { artificial flavors, sucralose, potassium sorbate, } \\
\text { sodium benzoate, EDTA. }\end{array}$} & \multicolumn{3}{|c|}{$\begin{array}{l}\text { Other Ingredients: Purified water, natural and } \\
\text { artificial flavors, sucralose, potassium sorbate, } \\
\text { sodium benzoate, EDTA. }\end{array}$} \\
\hline
\end{tabular}




\subsection{Experimental Design}

Following parental consent and child assent, basic demographic information was collected and questionnaires were self-administered to collect information regarding physical activity levels, caffeine consumption habits, and medical history. Anthropometric measures including height, weight, waist circumference and body-fat determination via dual-energy X-ray absorptiometry were collected. Participants completed trials in a randomized and double-blind fashion, each trial separated by 1-4 weeks. Although diet was not strictly controlled, participants were instructed to record and consume the same meal prior to each trial. Participants also arrived at the laboratory following a $24 \mathrm{~h}$ abstention from caffeine and vigorous exercise and after an overnight fast (10-12 h) to undergo an oral glucose tolerance test (OGTT). Upon arrival, participants were given a brief questionnaire to screen for conditions that may exclude them from the blood draw. After confirming compliance, an indwelling catheter was inserted into an antecubital vein and a fasting blood sample was collected. Immediately after, the treatment drinks: DECAF or CAF were consumed. Trial order was randomly assigned by a computer-generated randomizing program. However, we did not control for menstrual cycle phase as previous work has shown that menstrual cycle has no effect on the absorption, distribution, metabolism and elimination of caffeine [16]. Participants, research assistants and the research nurse performing the OGTT were blinded throughout the trial. Following treatment, subjects waited for $40 \mathrm{~min}$ before a second blood sample was taken (time: $0 \mathrm{~min}$ ), immediately followed by the ingestion of a glucose drink ( $1.75 \mathrm{~g} / \mathrm{kg}$ body weight Trutol, to a maximum of $75 \mathrm{~g})$. Additional blood samples were drawn at 30, 45, 60, 90 and 120 min. Data on participant characteristics and the DECAF trial have been previously reported [17] and are included here to provide context to the novel data on CAF administration. In this study, characteristics of males and females are reported separately.

\subsection{Blood Analysis}

At each time point, blood was collected in a vacutainer treated with EDTA (BD Vacutainer ${ }^{\circledR}$ ) and placed on ice immediately for later glucose analysis via the glucose oxidase method using a colorimetric assay (Cayman Chemical Company, Ann Arbor, MI, USA). In addition, EDTA tubes were treated with diprotinin-A (0.034 g/L blood; MP Biomedicals), sigma protease inhibitor (1 g/L blood; Sigma-Aldrich, St. Louis, MO, USA) and Roche Pefabloc ( $1 \mathrm{~g} / \mathrm{L}$ of blood) for analysis of gut and metabolic hormones. After blood collection, samples were centrifuged and the supernatant was collected and frozen at $-80{ }^{\circ} \mathrm{C}$ for later determination. Metabolic-related hormones were simultaneously quantified by a multiplex assay (Millipore, St. Charles, MO, USA) according to the manufacturer's protocol. Analytes included C-Peptide, ghrelin, glucose-dependent insulinotropic peptide (GIP), glucagon-like peptide-1 (GLP-1) (active), glucagon, insulin, leptin and pancreatic polypeptide YY (PYY) (total). The assay sensitivities of these markers ranged from $0.6-87 \mathrm{pg} / \mathrm{mL}$.

\subsection{Combined Caffeine Sensitivity Allele Score}

All participants ejected $5 \mathrm{~mL}$ of saliva into a tube for genetic analysis (Oragene, DNA Genotek, Ottawa, ON, Canada). Immediately following collection, tubes were sealed and stored until shipment. Analyses were run on data collected before December 2016. DNA extraction and genotyping were performed on saliva samples by an accredited laboratory (Laboratory Corporation of America). Comprehensive genotyping (933 202 SNP Chip, Illumina OmniExpress Plus Genotyping Bead Chip, 23andMe, Sunnyvale, CA, USA) was conducted as per manufacturer standard [18]. Five analytically validated variants involved in caffeine metabolism (rs4410790, rs2470893, rs2472297, rs2472299 and rs762551) were selected for analysis based on previous methods [19].

\subsection{Serum Metabolomics Analysis}

To gain insight into metabolic differences arising from either slow or fast caffeine metabolism, metabolomics analysis was performed on 39 serum samples collected at $120 \mathrm{~min}$ of each OGTT. A single 
sample was not able to be assessed due to volume restrictions. Samples were analyzed as previously described by ${ }^{1} \mathrm{H}-\mathrm{NMR}$ spectroscopy [20]. Briefly, serum samples were filtered in Amicon $10 \mathrm{kDa}$ cutoff filters (Millipore, Billerica, MA, USA). Four hundred microliters of filtered serum was mixed with $200 \mu \mathrm{L}$ phosphate buffer and $50 \mu \mathrm{L}$ deuterated water and measured by means of $1 \mathrm{D}{ }^{1} \mathrm{H}-\mathrm{NMR}$ spectroscopy on an Avance II $600 \mathrm{MHz}$ spectrometer (Bruker BioSpin, Milton, ON, Canada). Data were analyzed in R 3.5.1 (R Foundation for Statistical Computing, Vienna, Austria). Spectra were split into bins of $0.01 \mathrm{ppm}$ width in the region between 9.5 and $0.5 \mathrm{ppm}$, and bin size was manually increased for signals that visibly changed positions between spectra. After removal of water and noise areas, 472 signals were left for analysis. Signal intensities were scaled by the probabilistic quotient normalization (PQN) method. General linear models (GLM) were calculated individually for each signal, using caffeine (DECAF/CAF) and caffeine metabolism score (slow/fast) to model the observed metabolite concentration. Adding an interaction term (caffeine: caffeineMetabolism) did not reveal any metabolites significantly changing effect direction through such interactions; therefore, this term was not used for further analysis. Resulting $p$-values were corrected using false discovery rate controlling at an FDR level of 20\%. Metabolites were identified using database queries (https://hmdb.ca/, https://bmrb.io/) and measurements of pure standard compounds.

\subsection{Statistical Analysis}

All other analyses were done using GraphPad Prism 7 (San Diego, CA, USA). Descriptive statistics are presented as mean and standard deviation (SD) for numerical/continuous variables and percentages for categorical variables. Area under the curve (AUC) for serial measurements of glucose and gut and metabolic hormones during the two hour OGTTs (time: 0-120 min) was calculated using the trapezoidal method [21]. Repeated measures ANOVA for parametric data and Friedman's test for non-parametric data were employed. If data violated the assumptions of sphericity, a Greenhouse-Geisser correction was utilized. Where significant treatment effects were found, a Sidak multiple hypothesis test identified which treatments were significantly affected. Paired $t$-tests were applied to explore if the insulin sensitivity index [22], insulin and glucose AUC differed between DECAF and CAF treatments. To examine the genetic influence on glucose response, an allele score was calculated for each participant as previously described by Nordestgaard et al. [19]. For each genotype, scores of 0,1 and 2 were assigned and further binned into either low (slow caffeine metabolism) (1-5) or high (fast caffeine metabolism) (6-10) categories. A two-way ANOVA and Sidak multiple hypothesis test was used to examine differences between these groups. Where applicable, residuals of the robust fit were analyzed to identify outliers. This method uses an outlier test adapted from the false discovery rate approach of testing for multiple comparisons using the ROUT method of regression set at $\mathrm{Q}=1 \%$ [23]. $p<0.05$ was considered significant.

\section{Results}

\subsection{Participant Characteristics}

A total of twenty adolescents (10 male, 10 female) participated in this study with a self-reported Tanner stage of $4.3 \pm 0.8$ (mean $\pm \mathrm{SD}$ ) (Table 2). No significant effects of sex, age or BMI on glucose or insulin AUC were found $(p>0.05)$; therefore, data from all participants were pooled. All fasting blood glucose and insulin levels were within normal ranges and there were no differences in baseline samples between treatments $(p>0.05)$. All participants reported exercising regularly with $40 \%$ of participants exercising 2-4 days a week and the remaining $60 \%$ exercising 5-7 days a week. The majority of participants $(n=17,85 \%)$ reported that they consumed caffeine. 
Table 2. Participant characteristics.

\begin{tabular}{lll}
\hline & Males & Females \\
\hline$n$ & 10 & 10 \\
Age (years) & $16.4 \pm 2.2$ & $17.5 \pm 2.2$ \\
Height (m) & $1.75 \pm 0.1$ & $1.64 \pm 0.1$ \\
Weight (kg) & $72.3 \pm 24.6$ & $59.3 \pm 4.3$ \\
BMI & $23.1 \pm 6.0$ & $22.2 \pm 2.2$ \\
BP (Systolic) & $133 \pm 15$ & $111 \pm 8$ \\
BP (Diastolic) & $71 \pm 9$ & $62 \pm 9$ \\
\% Fat & $17.3 \pm 4.7$ & $24.2 \pm 4.5$ \\
\% Lean & $79.4 \pm 4.3$ & $72.4 \pm 4.4$ \\
\hline are shown separately for males and females (mean \pm SD).
\end{tabular}

\subsection{Impact of Caffeine-Containing Energy Shots on Glucose and Insulin Responses}

Glucose and insulin OGTT curves are shown in Figure 1. Compared to DECAF, significant increases in glucose concentrations were observed with CAF at 30, 45, 60 and 120 min during the OGTT $(p<0.05$, Figure 1A). Examination of mean AUC showed consumption of CAF resulted in a $25 \%$ increase in glucose excursion with values of $556.9 \pm 26.8$ and $683.8 \pm 31.4\left(\mathrm{mmol} / \mathrm{L}^{*} 120 \mathrm{~min}^{-1}\right)$ for DECAF and CAF, respectively $(p<0.0001)$ (Figure $1 \mathrm{~A})$. When individual responses were compared between DECAF and CAF treatments (AUC), the majority of participants (18/20) showed an exaggerated glucose response when caffeine was consumed (Figure 1B). Insulin levels were not significantly different at any time point examined $(p>0.05)$ (Figure 1C). However, the mean AUC was greater following CAF treatment with a $26 \%$ increase in insulin excursion with values of 42,437.2 \pm 4711.1 and 52,324.5 \pm 7371.2 $\mathrm{pmol} / \mathrm{L}^{*} 120 \mathrm{~min}^{-1}$ for DECAF and CAF, respectively ( $p=0.037$ ) (Figure 1D). Analysis of individual change between DECAF and CAF revealed larger inter-individual variation with 15/20 participants showing an increase in overall insulin responses based on AUC (Figure 1D) with CAF. The insulin sensitivity index (ISI) [22] was also affected by treatment (5.66 $\pm 0.48,4.62 \pm 0.45$, for DECAF and CAF, $p=0.0016)$ and was significantly lower following CAF treatment, indicating insulin resistance due to CAF consumption (Figure 1E). Presence or absence of caffeine was confirmed by a serum sample obtained at $120 \mathrm{~min}$. Results showed a concentration of $0.31 \pm 0.5 \mu \mathrm{mol} / \mathrm{L}$ and $22.9 \pm 1.5 \mu \mathrm{mol} / \mathrm{L}$ for DECAF and CAF trials, respectively $(p<0.001)$ (Figure 1F). 
A

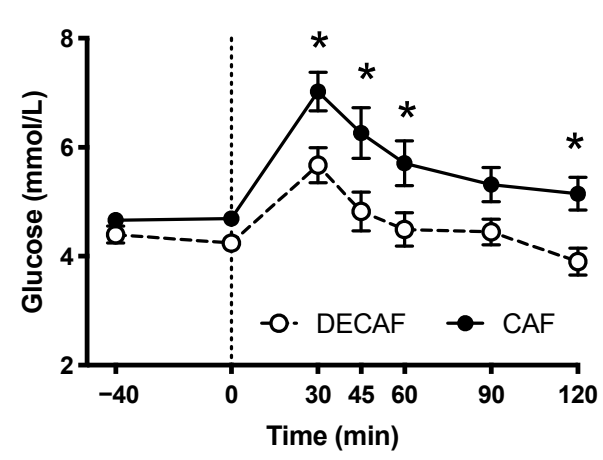

C

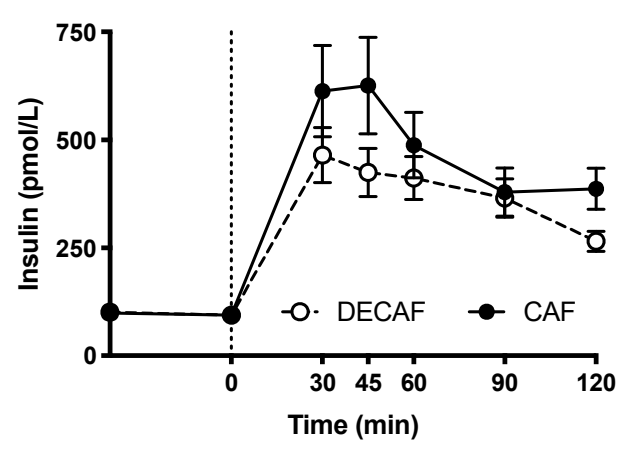

$\mathbf{E}$

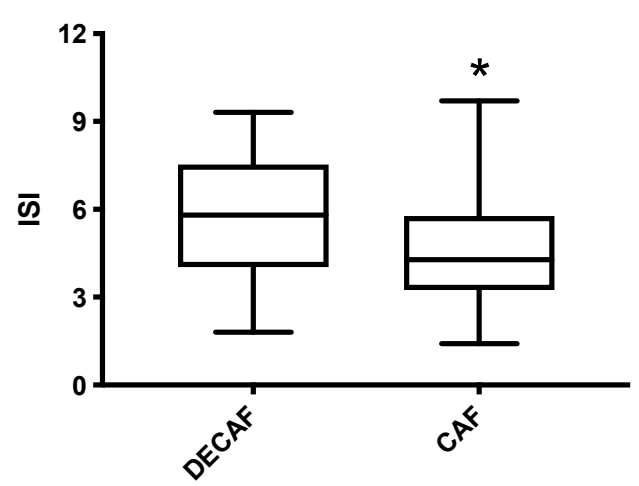

B

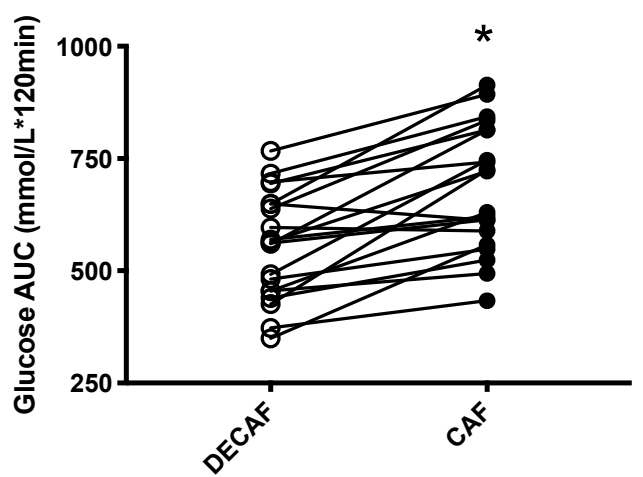

D

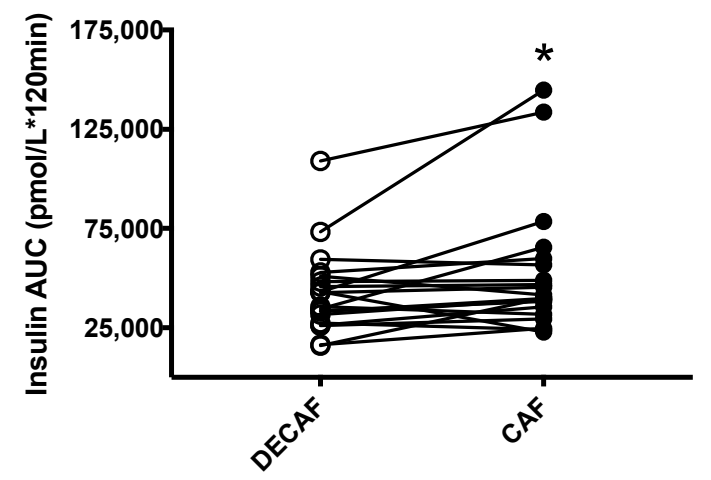

$\mathbf{F}$

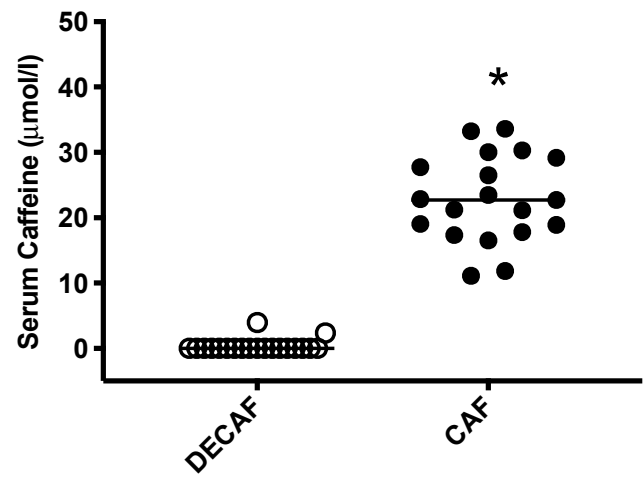

Figure 1. Postprandial glucose and insulin responses following ingestion of either a decaffeinated (DECAF) or caffeine-containing (CAF, $5 \mathrm{mg} / \mathrm{kg}$ ) energy shot at $-40 \mathrm{~min}$ followed by an oral glucose tolerance test (OGTT) at 0 min (indicated by a dashed line). (A) Blood glucose excursion curves. (B) Individual changes in glucose area under the curve calculated by the trapezoidal method (AUC). (C) Insulin excursion curve. (D) Individual changes in insulin AUC. (E) Calculated Matsuda insulin sensitivity index (ISI) [22] for each treatment (95\% confidence interval). (F) Mean serum caffeine concentrations obtained at $120 \mathrm{~min}$ of each OGTT. All data are shown as means $\pm \mathrm{SE}, n=20$ subjects in a randomized, cross-over design. * Indicates $p<0.05$, a statistically significant difference between treatments.

\subsection{Impacts of Caffeine-Containing Energy Shots on Gut and Metabolic Hormone Secretion}

A summary of all hormone results are presented in Table 3. No differences were noted between CAF and DECAF treatments for glucagon, leptin, PYY, ghrelin, GIP or GLP-1 $(p>0.05)$. 
Table 3. Gut and metabolic hormones area under the curve (AUC) response to treatments.

\begin{tabular}{|c|c|c|c|}
\hline Measure & Treatment & AUC Mean (SE) & $p$-Value \\
\hline Ghrelin (pg/mL/120 min) & $\begin{array}{l}\text { DECAF } \\
\text { CAF }\end{array}$ & $\begin{array}{l}5208.2(519.2) \\
5173.6(615.3)\end{array}$ & $p=0.945$ \\
\hline PYY (pg/mL/120 min) & $\begin{array}{l}\text { DECAF } \\
\text { CAF }\end{array}$ & $\begin{array}{l}7530.1(868.4) \\
7771.1(864.2)\end{array}$ & $p=0.412$ \\
\hline Leptin (pg/mL/120 min) & $\begin{array}{l}\text { DECAF } \\
\text { CAF }\end{array}$ & $\begin{array}{l}536,471.8(72,667.4) \\
489,282.6(67,250.3)\end{array}$ & $p=0.246$ \\
\hline $\mathrm{GIP}(\mathrm{pg} / \mathrm{mL} / 120 \mathrm{~min})$ & $\begin{array}{l}\text { DECAF } \\
\text { CAF }\end{array}$ & $\begin{array}{l}2960.6(565.8) \\
3003.4(517.0)\end{array}$ & $p=0.915$ \\
\hline GLP-1(pmol/L/120 min & $\begin{array}{l}\text { DECAF } \\
\text { CAF }\end{array}$ & $\begin{array}{l}407.0(47.8) \\
383.2(40.4)\end{array}$ & $p=0.438$ \\
\hline Glucagon (ng/L/120 min) & $\begin{array}{l}\text { DECAF } \\
\text { CAF }\end{array}$ & $\begin{array}{l}3085.8(508.1) \\
2963.2(347.7)\end{array}$ & $p=0.998$ \\
\hline C-peptide (nmol/L/120 min) & $\begin{array}{l}\text { DECAF } \\
\text { CAF }\end{array}$ & $\begin{array}{l}115.0(7.2) \\
120.1(6.7)\end{array}$ & $p=0.241$ \\
\hline
\end{tabular}

Values for each trial, decaffeinated 5-h energy (DECAF) and caffeinated 5-h energy (CAF) and their corresponding $p$-values are shown. Abbreviations are as follows: Gastric inhibitory polypeptide, GIP; Glucagon-like peptide-1, GLP-1; Peptide YY, PYY.

\subsection{Combined Caffeine Sensitivity Allele Score}

Due to the limited and underpowered sample size of this study, genetic variants of interest were analyzed as a composite allele score [19]. Briefly, five genetic variants combined into an allele score yielding scores between 0 and 10. Scores were then binned into either low (slow caffeine metabolism, 1-5) or high (fast caffeine metabolism, 6-10) for analysis. In total, 9 participants were classified as having a low allele score, while the remaining 11 subjects were classified as having a high score. Details of the analysis and variants present in participants are shown in Table 4. No differences in baseline glucose or insulin levels were found between slow and fast allele scores prior to the CAF and DECAF trials ( $p>0.05$, data not shown). Results of this analysis showed individuals with a high allele score experienced greater glucose intolerance as shown by significant differences in glucose and insulin excursion as well as a trend in the ISI with $p=0.07$ (Figure 2). In contrast, only glucose excursion was different between CAF and DECAF treatments in those individuals with a low allele score. This difference appears to be associated with insulin secretion and peak insulin levels that were greater in high vs. low allele score individuals ( $p<0.05$, data not shown).

A

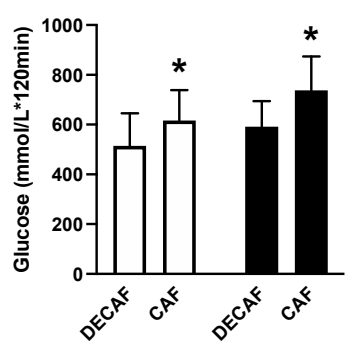

B

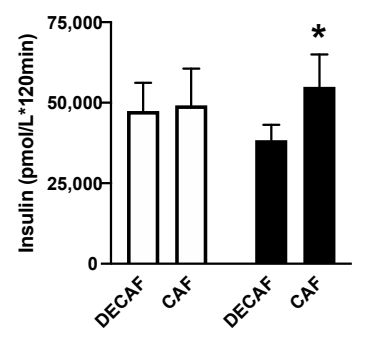

C

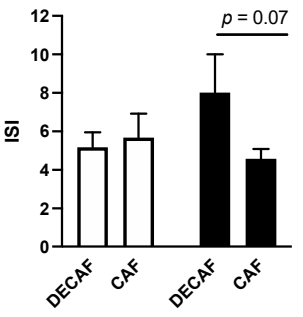

Slow Metabolism

Fast Metabolism

Figure 2. Genetic influences of caffeine-containing energy shot consumption on glucose and insulin tolerance. Subjects were classified as having either a low (slow caffeine metabolism, $n=9$ ) or high (fast caffeine metabolism, $n=11$ ) allele score based on variation at rs762551, rs4410790, rs2470893, rs2472297 and rs2472299 as described by Nordestgaard [19]. (A) Blood glucose AUC, (B) Insulin AUC, (C) Matsuda insulin sensitivity index (ISI) [22] in participants classified with slow or fast caffeine metabolism. All data are shown as means $\pm \mathrm{SE}, n=20$ subjects in a randomized, cross-over design. ${ }^{*}$ Indicates $p<0.05$, a statistically significant difference between treatments. 
Table 4. Combined Caffeine Sensitivity Allele Score.

\begin{tabular}{|c|c|c|c|c|c|c|c|}
\hline SNP & GENE & CHR & POSITION & GMAF & ALLELE & SCORE & $\overline{\text { COUNT }}$ \\
\hline \multirow{3}{*}{ rs4410790 } & \multirow{3}{*}{$A H R$} & \multirow{3}{*}{13} & \multirow{3}{*}{17284577} & \multirow{3}{*}{$\mathrm{C}>\mathrm{T}, 0.387$} & TT & 0 & 2 \\
\hline & & & & & $\mathrm{CT}$ & 1 & 11 \\
\hline & & & & & $\mathrm{CC}$ & 2 & 7 \\
\hline \multirow{3}{*}{ rs2470893 } & \multirow{3}{*}{ CYP1A1-4011 } & \multirow{3}{*}{15} & \multirow{3}{*}{75019449} & \multirow{3}{*}{$\mathrm{C}>\mathrm{T}, 0.278$} & $\mathrm{CC}$ & 0 & 9 \\
\hline & & & & & TC & 1 & 10 \\
\hline & & & & & TT & 2 & 1 \\
\hline \multirow{3}{*}{ rs2472297 } & \multirow{3}{*}{ CYP1A1-12441 } & \multirow{3}{*}{15} & \multirow{3}{*}{75027880} & \multirow{3}{*}{$C>\mathrm{T}, 0.225$} & $\mathrm{CC}$ & 0 & 12 \\
\hline & & & & & $\mathrm{TC}$ & 1 & 8 \\
\hline & & & & & $\mathrm{TT}$ & 2 & 0 \\
\hline \multirow{3}{*}{ rs2472299 } & \multirow{3}{*}{ CYP1A1 } & \multirow{3}{*}{15} & \multirow{3}{*}{74741059} & \multirow{3}{*}{$G>A, 0.350$} & AA & 0 & 3 \\
\hline & & & & & GA & 1 & 5 \\
\hline & & & & & GG & 2 & 12 \\
\hline \multirow{3}{*}{ rs762551 } & \multirow{3}{*}{ CYP1A2-163 } & \multirow{3}{*}{15} & \multirow{3}{*}{75041917} & \multirow{3}{*}{$A>C, 0.674$} & $\mathrm{CC}$ & 0 & 3 \\
\hline & & & & & $\mathrm{AC}$ & 1 & 5 \\
\hline & & & & & AA & 2 & 12 \\
\hline
\end{tabular}

A total of five alleles were used to calculate a combined score as previously described [19]. Low allele score (1-5) represents combination of genotypes associated with slow caffeine metabolism; high allele score represents combination of genotypes contributing to fast caffeine metabolism (6-10). Count (far right column) represents the genotypes of the participants. Abbreviations are as follows: aryl hydrocarbon receptor (AHR); chromosome (Chr); cytochromes P450 (CYP); minor allele frequency (MAF); single nucleotide polymorphism (SNP).

\subsection{Serum Metabolomics}

General linear model (GLM) analysis revealed strong and significant metabolic impacts of caffeine consumption. This is visualized in Figure 3A,B by principal component analysis (PCA) of significant metabolites, showing clear separation between DECAF vs CAF treatments as well as between slow and fast caffeine metabolizers. A PCA analysis of the full dataset can be found in Figure S1. Specifically, serum caffeine, 1,7-dimethylxanthine, folic acid and lactic acid were positively correlated to caffeine intake, while serum tartaric acid and choline decreased with caffeine intake (Figure 3C,D, Table S1).

Consistent with our metabolic results, serum glucose levels were elevated in fast vs. slow metabolizers with CAF. Levels of circulating caffeine as well as its primary metabolite 1,7-dimethylxanthine (paraxanthine) were found to be significantly higher in individuals with fast metabolism scores. Other distinct metabolites included citric acid that was higher in fast metabolizers, while proline levels were decreased in this group. 


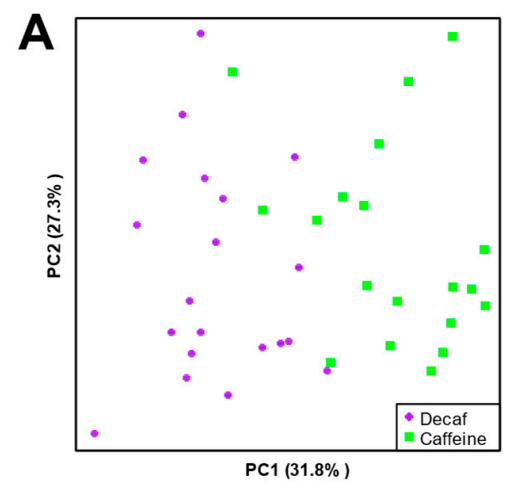

B

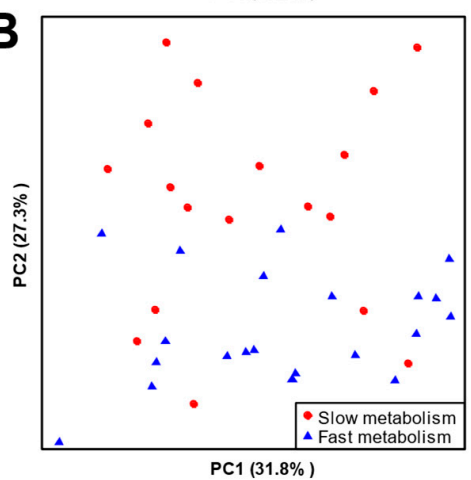

C

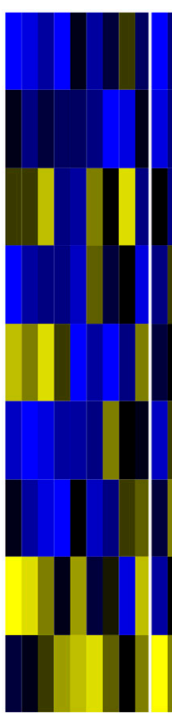

Slow

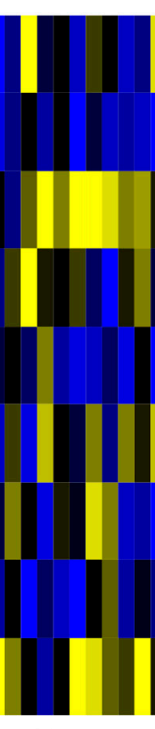

Fast Decaf

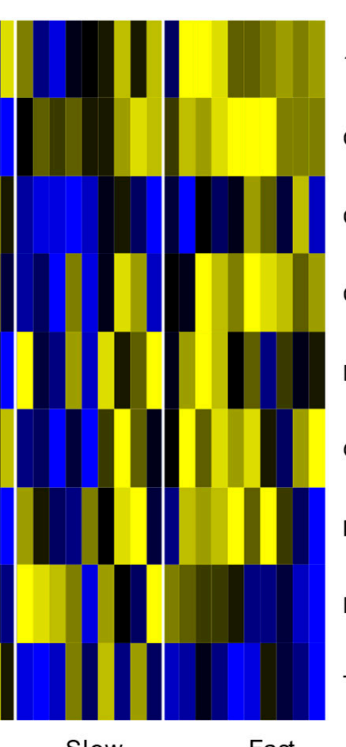

Slow Caffeine
1,7-Dimethy Ixanthine

Caffeine

Choline

Citric acid

Folic acid

Glucose

Lactic acid Tartaric acid

Fast

\begin{tabular}{|l|c|c|c|c|}
\hline \multirow{2}{*}{\multicolumn{1}{|c|}{ Metabolite }} & \multicolumn{2}{|c|}{$p$-values } & \multicolumn{2}{c|}{ Coefficients } \\
\cline { 2 - 5 } & $\begin{array}{c}\text { Slow vs. Fast Caffeine } \\
\text { Metabolism }\end{array}$ & DECAF vs. CAF & $\begin{array}{c}\text { Slow vs. Fast Caffeine } \\
\text { Metabolism }\end{array}$ & DECAF vs. CAF \\
\hline 1,7-Dimethylxanthine & 0.017 & 0.002 & + & + \\
Caffeine & 0.133 & $4.02 \times 10^{-16}$ & + & + \\
Choline & 0.009 & $3.19 \times 10^{-4}$ & + & + \\
Citric acid & 0.002 & 0.031 & + & + \\
Folic acid & 0.255 & 0.006 & + & + \\
Glucose & 0.002 & 0.023 & + & + \\
Lactic acid & 0.366 & 0.004 & - & + \\
Proline & $7.13 \times 10^{-4}$ & 0.253 & + & + \\
Tartaric acid & 0.499 & $3.24 \times 10^{-5}$ & & + \\
\hline
\end{tabular}

Figure 3. Serum metabolomics $\left({ }^{1} \mathrm{H}-\mathrm{NMR}\right)$ of $120 \mathrm{~min}$ time point of each OGTT. (A) Principal component analysis (PCA) showing separation of DECAF and CAF treatments. (B) PCA analysis showing separation of slow and fast allele scores. (C) Heatmap depicting significant changes between both DECAF and CAF treatments as well as slow and fast allele scores. (D) Table of serum metabolites significantly correlating with caffeine intake and/or caffeine metabolism score along with their $p$-values and direction (either + or - ) of coefficient change.

\section{Discussion}

Caffeine-containing energy shots represent a new and relatively understudied area of nutrition [24]. In the present study we evaluated the metabolic impact of these small format beverages in adolescents, who represent key consumers of the products [25]. Very little is known about the metabolic effects of caffeine energy shot consumption in adolescents who by nature of the normal influences of puberty are generally more insulin resistant [26]. Our results show CAF consumption, equivalent to one sugar-free small format energy shot containing $\sim 5 \mathrm{mg} / \mathrm{kg}$ caffeine, induces insulin resistance in adolescents compared to a control, DECAF beverage. Specifically, caffeine resulted in a $\sim 25 \%$ increase in glucose and insulin excursion. Insulin resistance resulting from caffeine consumption was also evident in examination of the ISI [22] that calculates whole body insulin sensitivity, through OGTT-derived glucose and insulin levels.

Our results are in line with other studies in adults showing that co-administration of alkaloid caffeine $[10,11,27,28]$ or caffeinated coffee [29-32] in combination with a carbohydrate load causes 
acute insulin resistance. However, the response to caffeine in coffee is attenuated compared to caffeine alone, presumably due to the presence of polyphenols and other coffee-related constituents $[33,34]$. This response is dose-dependent [13] and occurs independently of previous caffeine exposure [14]. Analysis of the impact of caffeine on glucose tolerance shows that on average, caffeine impairs glucose tolerance (evaluated by either an oral glucose tolerance test or insulin clamp) in the range of 30\% [6]. Numerous theories as to the mechanism by which caffeine impairs glucose tolerance at physiological concentrations have been proposed. Among these, the antagonism of adenosine receptors by caffeine (a potent, non-specific adenosine receptor antagonist) predominates, with evidence showing that caffeine directly impacts both insulin and exercise-stimulated glucose uptake in skeletal muscle [35-37].

In the present study, the energy shots were commercially available preparations in CAF and DECAF formats. The ingredient profile of the two beverages was similar, mainly differing in their caffeine concentration. This study design allowed the impact of caffeine in energy shots to be determined, but the impact of other energy shot ingredients should not be overlooked. Basrai and colleagues [38] conducted an elegant study wherein the impact of a large volume $(1000 \mathrm{~mL})$, caffeine-containing energy drink was studied on both cardiovascular and metabolic responses in young adults. Results demonstrated adverse impacts of the energy drink on blood pressure, prolongation of the corrected QT interval (QTc) interval as well as insulin sensitivity [38]. The caffeinated energy drink consisting of $32 \mathrm{mg} / 100 \mathrm{~mL}$ caffeine, $400 \mathrm{mg} / 100 \mathrm{~mL}$ taurine and $31 \mathrm{mg} / 100 \mathrm{~mL}$ glucuronolactone resulted in elevations in insulin that caused a greater modified homeostatic model assessment of insulin resistance (HOMA-IR) score, indicating greater insulin resistance. Of note, changes in HOMA-IR resulting from energy drink consumption in the study were greater than caffeine administration alone, as was the QTc interval prolongation [38]. This highlights a potentially synergistic impact of energy drink ingredients, an issue that has been previously raised in the energy drink literature and may lead to more adverse effects than traditional caffeinated beverages such as coffee [39-41].

As an individual's preference, sensitivity and metabolism of caffeine are relatively stable over a lifetime and determined in part by genetics [42], we conducted a preliminary analysis to examine the role of genetic variation in caffeine-induced insulin resistance. Because this analysis was based on a small number of subjects, individuals were classified as having either a high or low allele score for variation of AHR, CYP1A1 and CYP1A2 genes (SNPs: rs762551, rs4410790, rs2470893, rs2472297 and rs2472299) as described by Nordestgaard [19]. Individuals with high allele scores, representative of fast caffeine metabolism, had significantly greater disruption to glucose and insulin secretion as well as impaired insulin sensitivity throughout the OGTT compared to individuals with a low allele score. A number of theories have been proposed to explain these findings. Keijzers et al. [43] theorize that tolerance to caffeine-induced insulin resistance may occur with chronic consumption, but individuals who are fast metabolizers of caffeine may be unable to develop this tolerance due to the short caffeine half-life. In agreement, our metabolomics results show both caffeine and its metabolite 1,7-dimethylxanthine (paraxanthine) were present in greater levels compared to slow metabolizers with CAF treatment at 120 min of the OGTT.

Likewise, it is also possible that caffeine metabolites contribute to its impact on glucose intolerance. Caffeine is rapidly and primarily metabolized by liver CYP1A2 and CYP2E1 into its three related dimethylxanthines; 1,3-dimethylxanthine (theophylline, 11\%), 3,7-dimethylxanthine (theobromine, $4 \%$ ) and 1,7-dimethylxanthine (paraxanthine, 80\%), through a series of demethylation steps [44]. These metabolites are also metabolically active and can influence glucose tolerance. Employing hyperinsulinemic-hypoglycemic clamps, De Galan et al. [45] demonstrated that theophylline infusion $(2.8 \mathrm{mg} / \mathrm{kg})$ causes profound insulin resistance as shown by a reduction in glucose infusion rates in both healthy controls and type 1 diabetes. Given this, the greater concentrations of caffeine metabolites in those with fast metabolism may explain in part why their glucose tolerance was affected to a greater extent with CAF consumption. Taken together, results of our study suggest that fast caffeine metabolizers who consume CED on a regular basis likely experience greater impairment in glucose tolerance compared to slow metabolizers. As such, it is likely that the $\sim 30 \%$ decline in insulin sensitivity 
seen with caffeine consumption in adults across dozens of studies minimizes the true impact of caffeine in this subgroup. Our overall finding that caffeine-induced glucose tolerance is affected by an individual's genetics adds to a growing body of evidence showing that caffeine preference, ergogenic potential, impacts on sleep and even disease-related susceptibility are genetically influenced $[15,46]$.

Several strengths and limitations need to be considered in the present study. A key strength is the examination of adolescents and commercially available energy shots in relation to glucose tolerance. In this study, the beverage was administered as a mixture and was sugar-free followed by a carbohydrate load (OGTT). While caffeine and the carbohydrate load were administered separately in our study to examine glucose tolerance, the majority of energy drinks (as opposed to small volume shots) are consumed as sugar-sweetened mixtures. It must also be acknowledged that the results from one beverage may not be applicable across the entire CAF beverage category given the wide range of ingredients and caffeine concentrations that are available. It is also likely that there are interactions between ingredients. In particular, concerns regarding an interaction between caffeine and taurine found in CAF have been highlighted in previous reports $[47,48]$.

Limitations include venous blood sampling (vs. arterial), lack of a standardized diet prior to trials as well as the administration of an OGTT that does not reflect what happens in everyday life where mixed meals are consumed. Additional limitations include the genetic analysis, which is based on a small number of subjects and a combined allele score. These results are preliminary and require confirmation at the individual genotype level. However, strong separation of slow and fast allele scores by metabolomics analyses adds strength to the applicability and validity of the approach. Next, all participants were healthy and within normal body mass ranges. Therefore, the clinical relevance of acute insulin resistance caused by caffeine in this demographic is unknown. It is very likely that responses are exaggerated in adolescents with obesity and/or insulin resistance.

\section{Conclusions}

CED are popular and frequently consumed among children and adolescents, an impressionable and understudied population. In the present study, we provide evidence that caffeine administered as part of a complex energy shot mixture causes acute, transient insulin resistance in adolescents. The clinical relevance of this finding warrants further investigation.

Supplementary Materials: The following are available online at http://www.mdpi.com/2072-6643/12/12/3850/s1, Figure S1: Serum metabolomics ( ${ }^{1} \mathrm{H}-\mathrm{NMR}$ ) of $120 \mathrm{~min}$ time point of each OGTT, Table S1: Serum compounds significantly correlating with caffeine intake (DECAF vs. CAF) or caffeine metabolism score (Low vs. High).

Author Contributions: Conceptualization, J.S., R.A.R., H.J.V.; methodology, J.S.; formal study and analysis, J.S., D.S.H., M.A.G., M.S.K.; all authors were involved in the preparation, writing and editing of the original draft preparation. All authors have read and agreed to the published version of the manuscript.

Funding: The research was funded by the Alberta Children's Hospital Research Institute, the National Science and Engineering Council of Canada (JS), and the Foods for Health Discovery Theme.

Acknowledgments: The authors wish to acknowledge the assistance of the following individuals; Claire Gougeon, who is a pediatric nurse who inserted indwelling catheters, Heidi Virtanen who was responsible for day-to-day study conduct and Alberto Nettel-Aguirre for statistical assistance.

Conflicts of Interest: The authors declare no conflict of interest.

\section{References}

1. Heckman, M.A.; Sherry, K.; de Mejia, E.G. Energy drinks: An assessment of their market size, consumer demographics, ingredient profile, functionality, and regulations in the United States. Compr. Rev. Food Sci. Food Saf. 2010, 9, 303-317. [CrossRef]

2. Reid, J.L.; McCrory, C.; White, C.M.; Martineau, C.; Vanderkooy, P.; Fenton, N.; Hammond, D. Consumption of Caffeinated Energy Drinks Among Youth and Young Adults in Canada. Prev. Med. Rep. 2017, 5, 65-70. [CrossRef] [PubMed] 
3. Committee on Nutrition and the Council on Sports Medicine and Fitness. Pediatrics Sports Drinks and Energy Drinks for Children and Adolescents: Are They Appropriate? Pediatrics 2011, 127, 1182-1189. [CrossRef] [PubMed]

4. Bigard, A.X. Risks of energy drinks in youths. Arch. Pediatr. 2010, 17, 1625-1631. [CrossRef]

5. Seifert, S.M.; Schaechter, J.L.; Hershorin, E.R.; Lipshultz, S.E. Health effects of energy drinks on children, adolescents, and young adults. Pediatrics 2011, 127, 511-528. [CrossRef]

6. Shearer, J.; Graham, T.E. Performance effects and metabolic consequences of caffeine and caffeinated energy drink consumption on glucose disposal. Nutr. Rev. 2014, 72, 121-136. [CrossRef]

7. Calamaro, C.J.; Mason, T.B.A.; Ratcliffe, S.J. Adolescents living the 24/7 lifestyle: Effects of caffeine and technology on sleep duration and daytime functioning. Pediatrics 2009, 123. [CrossRef]

8. Owens, J.A.; Mindell, J.; Baylor, A. Effect of energy drink and caffeinated beverage consumption on sleep, mood, and performance in children and adolescents. Nutr. Rev. 2014, 72, 65-71. [CrossRef]

9. Graham, T.E.; Sathasivam, P.; Rowland, M.; Marko, N.; Greer, F.; Battram, D. Caffeine ingestion elevates plasma insulin response in humans during an oral glucose tolerance test. Can. J. Physiol. Pharmacol. 2001, 79, 559-565. [CrossRef]

10. Petrie, H.J.; Chown, S.E.; Belfie, L.M.; Duncan, A.M.; McLaren, D.H.; Conquer, J.A.; Graham, T.E. Caffeine ingestion increases the insulin response to an oral-glucose-tolerance test in obese men before and after weight loss. Am. J. Clin. Nutr. 2004, 80, 22-28. [CrossRef]

11. Robinson, L.E.; Spafford, C.; Graham, T.E.; Smith, G.N. Acute Caffeine Ingestion and Glucose Tolerance in Women with or without Gestational Diabetes Mellitus. J. Obstet. Gynaecol. Canada 2009, 31, 304-312. [CrossRef]

12. Lee, S.; Hudson, R.; Kilpatrick, K.; Graham, T.E.; Ross, R. Caffeine ingestion is associated with reductions in glucose uptake independent of obesity and type 2 diabetes before and after exercise training. Diabetes Care 2005, 28, 566-572. [CrossRef] [PubMed]

13. Beaudoin, M.-S.; Allen, B.; Mazzetti, G.; Sullivan, P.J.; Graham, T.E. Caffeine ingestion impairs insulin sensitivity in a dose-dependent manner in both men and women. Appl. Physiol. Nutr. Metab. 2013, 38, 140-147. [CrossRef] [PubMed]

14. Dekker, M.J.; Gusba, J.E.; Robinson, L.E.; Graham, T.E. Glucose homeostasis remains altered by acute caffeine ingestion following 2 weeks of daily caffeine consumption in previously non-caffeine-consuming males. $\mathrm{Br}$. J. Nutr. 2007, 98, 556. [CrossRef]

15. Nehlig, A. Interindividual differences in caffeine metabolism and factors driving caffeine consumption. Pharmacol. Rev. 2018. [CrossRef] [PubMed]

16. McLean, C.; Graham, T.E. Effects of exercise and thermal stress on caffeine pharmacokinetics in men and eumenorrheic women. J. Appl. Physiol. 2002, 93, 1471-1478. [CrossRef]

17. Mayengbam, S.; Virtanen, H.; Hittel, D.S.; Elliott, C.; Reimer, R.A.R.A.; Vogel, H.J.H.J.; Shearer, J. Metabolic consequences of discretionary fortified beverage consumption containing excessive vitamin B levels in adolescents. PLoS ONE 2019, 14. [CrossRef]

18. Carere, D.A.; Couper, M.P.; Crawford, S.D.; Kalia, S.S.; Duggan, J.R.; Moreno, T.A.; Mountain, J.L.; Roberts, J.S.; Green, R.C.; Krier, J.B.; et al. Design, methods, and participant characteristics of the Impact of Personal Genomics (PGen) Study, a prospective cohort study of direct-to-consumer personal genomic testing customers. Genome Med. 2014, 6, 96. [CrossRef]

19. Nordestgaard, A.T.; Thomsen, M.; Nordestgaard, B.G. Coffee intake and risk of obesity, metabolic syndrome and type 2 diabetes: A Mendelian randomization study. Int. J. Epidemiol. 2015, 44, 551-565. [CrossRef]

20. Gronwald, W.; Klein, M.S.; Kaspar, H.; Fagerer, S.R.; Nürnberger, N.; Dettmer, K.; Bertsch, T.; Oefner, P.J. Urinary Metabolite Quantification Employing 2D NMR Spectroscopy. Anal. Chem. 2008, 80, 9288-9297. [CrossRef]

21. Yeh, K.C.; Kwan, K.C. A comparison of numerical integrating algorithms by trapezoidal, Lagrange, and spline approximation. J. Pharmacokinet. Biopharm. 1978. [CrossRef] [PubMed]

22. Matsuda, M.; DeFronzo, R.A. Insulin sensitivity indices obtained from oral glucose tolerance testing: Comparison with the euglycemic insulin clamp. Diabetes Care 1999, 22, 1462-1470. [CrossRef] [PubMed]

23. Motulsky, H.J.; Brown, R.E. Detecting outliers when fitting data with nonlinear regression-A new method based on robust nonlinear regression and the false discovery rate. BMC Bioinform. 2006, 7, 123. [CrossRef] [PubMed] 
24. Sorkin, B.C.; Coates, P.M. Caffeine-containing energy drinks: Beginning to address the gaps in what we know. Adv. Nutr. 2014, 5, 541-543. [CrossRef]

25. Hwang, S.B.; Park, S.; Jin, G.-R.; Jung, J.H.; Park, H.J.; Lee, S.H.; Shin, S.; Lee, B.-H. Trends in Beverage Consumption and Related Demographic Factors and Obesity among Korean Children and Adolescents. Nutrients 2020, 12, 2651. [CrossRef]

26. Goran, M.I.; Gower, B.A. Longitudinal Study on Pubertal Insulin Resistance. Diabetes 2001, 50, $2444-2450$. [CrossRef]

27. Robinson, L.E.; Savani, S.; Battram, D.S.; McLaren, D.H.; Sathasivam, P.; Graham, T.E. Caffeine ingestion before an oral glucose tolerance test impairs blood glucose management in men with type 2 diabetes. J. Nutr. 2004, 134, 2528-2533. [CrossRef]

28. Shi, X.; Xue, W.; Liang, S.; Zhao, J.; Zhang, X. Acute caffeine ingestion reduces insulin sensitivity in healthy subjects: A systematic review and meta-analysis. Nutr. J. 2016, 15, 1-8. [CrossRef]

29. Johnston, K.L.; Clifford, M.N.; Morgan, L.M. Coffee acutely modifies gastrointestinal hormone secretion and glucose tolerance in humans: Glycemic effects of chlorogenic acid and caffeine. Am. J. Clin. Nutr. 2003, 78, 728-733. [CrossRef]

30. Beaudoin, M.S.; Robinson, L.E.; Graham, T.E. An oral lipid challenge and acute intake of caffeinated coffee additively decrease glucose tolerance in healthy men. J. Nutr. 2011, 141, 574-581. [CrossRef]

31. Moisey, L.L.; Robinson, L.E.; Graham, T.E. Consumption of caffeinated coffee and a high carbohydrate meal affects postprandial metabolism of a subsequent oral glucose tolerance test in young, healthy males. Br. J. Nutr. 2010, 103, 833-841. [CrossRef] [PubMed]

32. Moisey, L.L.; Kacker, S.; Bickerton, A.C.; Robinson, L.E.; Graham, T.E. Caffeinated coffee consumption impairs blood glucose homeostasis in response to high and low glycemic index meals in healthy men. Am. J. Clin. Nutr. 2008, 87, 1254-1261. [CrossRef] [PubMed]

33. Battram, D.S.; Arthur, R.; Weekes, A.; Graham, T.E. The glucose intolerance induced by caffeinated coffee ingestion is less pronounced than that due to alkaloid caffeine in men. J. Nutr. 2006, 136, 1276-1280. [CrossRef] [PubMed]

34. Shearer, J.; Farah, A.; De Paulis, T.; Bracy, D.P.; Pencek, R.R.; Graham, T.E.; Wasserman, D.H. Quinides of Roasted Coffee Enhance Insulin Action in Conscious Rats. J. Nutr. 2003, 133. [CrossRef] [PubMed]

35. Vergauwen, L.; Hespel, P.; Richter, E.A. Adenosine receptors mediate synergistic stimulation of glucose uptake and transport by insulin and by contractions in rat skeletal muscle. J. Clin. Investig. 1994, 93, 974-981. [CrossRef] [PubMed]

36. Thong, F.S.L.; Graham, T.E. The putative roles of adenosine in insulin- and exercise-mediated regulation of glucose transport and glycogen metabolism in skeletal muscle. Can. J. Appl. Physiol. 2002, 27, 152-178. [CrossRef]

37. Fredholm, B.B. On the Mechanism of Action of Theophylline and Caffeine. Acta Med. Scand. 1985, 217, 149-153. [CrossRef]

38. Basrai, M.; Schweinlin, A.; Menzel, J.; Mielke, H.; Weikert, C.; Dusemund, B.; Putze, K.; Watzl, B.; Lampen, A.; Bischoff, S.C. Energy drinks induce acute cardiovascular and metabolic changes pointing to potential risks for young adults: A randomized controlled trial. J. Nutr. 2019, 149, 441-450. [CrossRef]

39. Higgins, J.P.; Yang, B.; Herrin, N.E.; Yarlagadda, S.; Le, G.T.; Ortiz, B.L.; Ali, A.; Infanger, S.C. Consumption of energy beverage is associated with attenuation of arterial endothelial flow-mediated dilatation. World J. Cardiol. 2017, 9, 162. [CrossRef]

40. Cotter, B.V.; Jackson, D.A.E.; Merchant, R.C.; Babu, K.M.; Baird, J.R.; Nirenberg, T.; Linakis, J.G. Energy drink and other substance use among adolescent and young adult emergency department patients. Pediatr. Emerg. Care 2013, 29, 1091-1097. [CrossRef]

41. Jackson, D.A.E.; Cotter, B.V.; Merchant, R.C.; Babu, K.M.; Baird, J.R.; Nirenberg, T.; Linakis, J.G. Behavioral and physiologic adverse effects in adolescent and young adult emergency department patients reporting use of energy drinks and caffeine. Clin. Toxicol. 2013, 51, 557-565. [CrossRef] [PubMed]

42. Laitala, V.S.; Kaprio, J.; Silventoinen, K. Genetics of coffee consumption and its stability. Addiction 2008, 103, 2054-2061. [CrossRef] [PubMed]

43. Keijzers, G.; De Galan, B.E.; Tack, C.J.; Smits, P. Caffeine can decrease insulin sensitivity in humans. Diabetes Care 2002, 25, 364-369. [CrossRef] [PubMed] 
44. Gu, L.; Gonzalez, F.J.; Kalow, W.; Tang, B.K. Biotransformation of caffeine, paraxanthine, theobromine and theophylline by cDNA-expressed human CYP1A2 and CYP2E1. Pharmacogenetics 1992, 2, 73-77. [CrossRef] [PubMed]

45. De Galan, B.E.; Tack, C.J.; Lenders, J.W.; Pasman, J.W.; Elving, L.D.; Russel, F.G.; Lutterman, J.A.; Smits, P. Theophylline improves hypoglycemia unawareness in type 1 diabetes. Diabetes 2002. [CrossRef] [PubMed]

46. Landolt, H.P. Genotype-Dependent Differences in Sleep, Vigilance, and Response to Stimulants. Curr. Pharm. Des. 2008, 14, 3396-3407. [CrossRef]

47. Higgins, J.P.; Tuttle, T.D.; Higgins, C.L. Energy beverages: Content and safety. Mayo Clin. Proc. 2010, 85, 1033-1041. [CrossRef]

48. Steinke, L.; Lanfear, D.E.; Dhanapal, V.; Kalus, J.S. Effect of "energy drink" consumption on hemodynamic and electrocardiographic parameters in healthy young adults. Ann. Pharmacother. 2009. [CrossRef]

Publisher's Note: MDPI stays neutral with regard to jurisdictional claims in published maps and institutional affiliations.

(C) 2020 by the authors. Licensee MDPI, Basel, Switzerland. This article is an open access article distributed under the terms and conditions of the Creative Commons Attribution (CC BY) license (http://creativecommons.org/licenses/by/4.0/). 